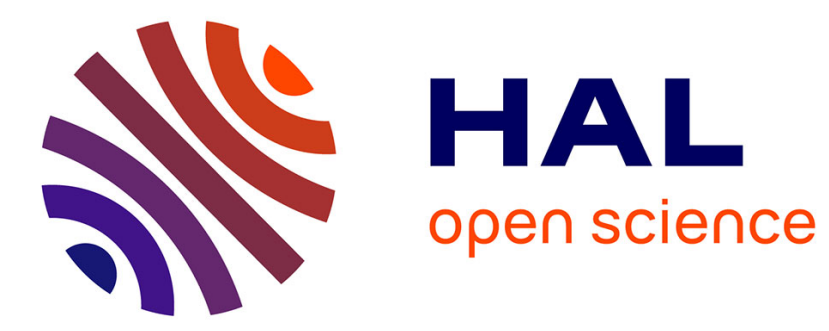

\title{
From spin flip excitations to the spin susceptibility enhancement of a two-dimensional electron gas
}

Florent Pérez, C. Aku-Leh, D. Richards, Bernard Jusserand, L. C. Smith, D. Wolverson, G. Karczewski

\section{- To cite this version:}

Florent Pérez, C. Aku-Leh, D. Richards, Bernard Jusserand, L. C. Smith, et al.. From spin flip excitations to the spin susceptibility enhancement of a two-dimensional electron gas. Physical Review Letters, 2007, 99 (2), pp.026403. 10.1103/PhysRevLett.99.026403 . hal-01287894

\section{HAL Id: hal-01287894 \\ https://hal.science/hal-01287894}

Submitted on 1 Jun 2016

HAL is a multi-disciplinary open access archive for the deposit and dissemination of scientific research documents, whether they are published or not. The documents may come from teaching and research institutions in France or abroad, or from public or private research centers.
L'archive ouverte pluridisciplinaire HAL, est destinée au dépôt et à la diffusion de documents scientifiques de niveau recherche, publiés ou non, émanant des établissements d'enseignement et de recherche français ou étrangers, des laboratoires publics ou privés. 


\title{
From spin flip excitations to the spin susceptibility enhancement of a two dimensional electron gas
}

\author{
F. Perez ${ }^{1}$, C. Aku-leh ${ }^{1,2}$, D. Richards ${ }^{2}$, B. Jusserand ${ }^{1}$, L. C. Smith ${ }^{3}$, D. Wolverson ${ }^{3}$, and G. \\ Karczewski $^{4}$ \\ ${ }^{1}$ Institut des NanoSciences de Paris, CNRS, Universités Paris 6 \& 7, 140 rue de Lourmel, \\ 75015 Paris, France \\ ${ }^{2}$ Department of Physics, King's College London, Strand, London WC2R 2LS, United \\ Kingdom \\ ${ }^{3}$ Department of Physics, University of Bath, Bath BA2 7AY, United Kingdom \\ ${ }^{4}$ Institute of Physics, Polish Academy of Science, Al. Lotnikow 32/46, 02-668 Warsaw, \\ Poland
}

\begin{abstract}
:
The g-factor enhancement of the spin-polarized two-dimensional electron gas was measured directly over a wide range of spin polarizations, using spin flip resonant Raman scattering spectroscopy on two dimensional electron gases embedded in $\mathrm{Cd}_{1-\mathrm{x}} \mathrm{Mn}_{\mathrm{x}} \mathrm{Te}$ semi-magnetic quantum wells. At zero Raman transferred momentum, the single particle spin flip excitation, energy $Z^{*}$, coexists in the Raman spectrum with the spin flip wave of energy Z, the bare giant Zeeman splitting. We compare the measured g-factor enhancement with recent spin susceptibility enhancement theories and deduce the spin polarization dependence of the mass renormalization.
\end{abstract}

PACS : 71.18.+y , 75.30.Ds, 78.30.-j, 75.50.Pp, 72.25.Dc 
It is well established that exchange and correlation Coulomb interactions at low electron densities are predicted to enhance the spin susceptibility $\chi$ of a paramagnetic electron gas over that of the Pauli spin susceptibility $\chi_{0}$ for non-interacting electrons ${ }^{1}$. Thus, a full characterization of this exchange-correlation enhancement of the spin susceptibility should underpin our understanding of spin resolved Coulomb interactions in homogeneous electron gases $^{2}$. There has been considerable recent interest in theoretical ${ }^{2,3,4}$ and experimental ${ }^{5,6,7,8,9}$ investigations of the spin susceptibility enhancement in low dimensional systems embedded in semiconductor heterostructures, aimed at finding evidence for the the spin susceptibility enhancement in very clean two dimensional electron gases (2DEGs) ${ }^{5,6}$, and for the eventual divergence of the spin susceptibility near the critical density for the Metal Insulator Transition observed in Si-MOSFET inversion layers ${ }^{7,8,9}$. However, these studies have not fully considered the dependence of the enhancement on the degree of spin polarization, although strong non linear behaviour is expected in a clean 2DEG.

The spin susceptibility enhancement is given by the relation ${ }^{10}$,

$$
\frac{\chi}{\chi_{0}}=\left(1+\frac{r_{s}^{2}}{2} \frac{\partial^{2} \varepsilon_{x c}}{\partial \zeta^{2}}\right)^{-1},
$$

where $\zeta=\left(n_{\uparrow}-n_{\downarrow}\right) /\left(n_{\uparrow}+n_{\downarrow}\right)$ is the spin polarization of an electron gas with equilibrium density $n_{2 D}=n_{\uparrow}+n_{\downarrow} \cdot r_{s}=\left(a_{B}^{*} \sqrt{\pi n_{2 D}}\right)^{-1}$ is the ratio of the mean spacing between electrons to the Bohr radius, $a_{B}^{*}$, and $\varepsilon_{x c}$ is the exchange-correlation part of the ground-state energy, expressed in Rydbergs ${ }^{11}$. 
Experimental investigations to date of clean 2DEGs in the metallic state have focused on the dependence of the spin-susceptibility enhancement on $r_{s}$. These have included thermodynamic measurements, for which the possible contribution of localized moments remains controversial, and magneto-transport measurements ${ }^{5,6,7,8}$, which are only sensitive to mobile electrons. The spin susceptibility enhancement factor is usually given by the relation,

$$
\chi / \chi_{0}=m^{*} g^{*} / m_{b} g_{b},
$$

where the electron mass $m^{*}$ and g-factor $g^{*}$ are renormalized relative to their respective noninteracting values determined at the conduction band minimum, $m_{b}$ and $g_{b}$. Magneto-transport measurements accurately determine the product of $m^{*}$ with $g^{*}$ in two ways: (i) Measurement of the in-plane field necessary to polarize fully the 2DEG $(|\zeta|=1)$; (ii) Determination of the specific tilt-angle of the field for which the energy spacing of Landau levels becomes identical to the spin splitting energy ${ }^{6,7,8}$. The Landau quantization introduced by the latter technique has to be kept negligible, and so only low spin polarization degrees can be probed $(|\zeta|<10 \%)$. The density dependence at $|\zeta|=1$ and $|\zeta|<0.1$ is now well understood; excellent agreement was obtained recently between experiment and theory using a quantum Monte Carlo (QMC) evaluation of the correlation energy and accounting for the finite thickness of the quantum well. However, the continuous spin-degree dependence of the spin-susceptibility enhancement has not been considered experimentally. Moreover, the g-factor enhancement $g^{*} / g_{b}$ is deduced after independent determinations of the mass $m^{*}$ using a multiple parameter fit and the product $\mathrm{m}^{*} g^{*}$, together with an assumed value for $m_{b}$ and $g_{b}{ }^{12}$. No direct measurement of the g-factor enhancement $g^{*} / g_{b}$ has yet been reported. 
Here, we provide a direct determination of $g^{*} / g_{b}$ over a wide range of spin polarization $(0<$ $|\zeta|<0.6$ ), by measuring directly and simultaneously both $g^{*}$ and $g_{b}$. From a comparison with the theories of Ref. 2 and 3, we extract data about the dependence of the mass enhancement on the spin polarization, which we then compare with recent predictions of a non-linear dependence of the spin-susceptibility and mass enhancement ${ }^{13}$ with spin-polarization.

We have employed electronic Raman scattering as a probe of electronic excitations of spinpolarized 2DEGs (SP2DEGs) embedded in dilute magnetic semiconductor (DMS) modulation-doped quantum wells. The giant Zeeman effect occurring in these systems allows the creation of a highly spin polarized electron gas with a very low magnetic field. This has the advantage of negligible Landau orbital quantization, contrary to GaAs-based systems. Indeed, beyond the immediate results presented here, we demonstrate that such DMS quantum wells provide an excellent model system for the study of the ideal spin-polarized electron gas.

Electronic Resonant Raman Scattering (RRS) is a powerful technique for measurement of the low energy excitation dispersions of the 2DEG Fermi disk, such as the determination of spin excitations in the quantum Hall regime ${ }^{14}$. We demonstrated recently that RRS may be employed as a probe of spin excitations dispersions in a SP2DEG embedded in a semimagnetic quantum well ${ }^{15}$. Here, we have investigated samples optimized so that the disorder introduced by random magnetic impurities has been reduced while conserving a sufficiently high giant Zeeman effect to achieve high spin polarization. Each sample comprises a $150 \AA$ wide $\mathrm{Cd}_{1-x} \mathrm{Mn}_{x}$ Te quantum well $(x=0.8 \pm 0.05 \%)$ and barriers of $\mathrm{Cd}_{0.8} \mathrm{Mg}_{0.2}$ Te with a spacer thickness of $200 \AA$ and different doping layer thicknesses in order to achieve electron densities $n_{2 D}$ in the range $2-3 \times 10^{11} \mathrm{~cm}^{-2}$ (the corresponding range for $r_{s}$ is 2.5 to 2.0). Measurements 
have been performed at $1.5 \mathrm{~K}$ in a backscattering Voigt configuration (see Fig. 1b) with inplane magnetic field below $4 \mathrm{~T}$, such that the minimum electron magnetic length remains comparable to the well width, rendering the magnetic orbital quantization negligible. Given this, the mass enhancement due to the magneto-hybrid band-bending is also negligible ${ }^{16}$.

The applied magnetic field polarizes localized spins of the Manganese ions which, through the exchange interaction with the conduction electrons, lift the spin degeneracy of the quantum well conduction band with a bare Zeeman energy given by,

$$
Z(B)=\bar{x} \cdot N_{0} \alpha \cdot\left\langle S_{z}(B, T)\right\rangle
$$

where $N_{0} \alpha=220 \mathrm{meV}$ is the ferromagnetic coupling integral between $s$ conduction electrons and $d$ Manganese electrons ${ }^{17} \cdot\left\langle S_{z}(B, T)\right\rangle$ is the thermal equilibrium average of the localized Manganese spins given by the modified Brillouin function and $\bar{x}$ is the paramagnetic Mn concentration $(\bar{x} \approx x)$. In a Fermi liquid, single-particle and collective excitations co-exist and the SP2DEG exhibits a spin flip wave (SFW) that involves a simultaneous flip of all electron spins. By Larmor's Theorem ${ }^{18}$, the zero wavevector SFW energy $\operatorname{SFW}(q=0)$ is sensitive only to the external magnetic field applied to the 2DEG. Here the coupling with Manganese spins plays the role of the external field, such that $S F W(q=0)=Z(B)$. In addition, a single electron flipping its spin sees its energy change by a renormalized Zeeman energy $Z^{*}(B)=Z(B)+W_{x c}$, due to exchange and correlation ${ }^{19}$. For the densities considered here, the ferromagnetic exchange dominates over correlations. As a consequence, flipping the spin of a single electron without disturbance of other spins is more costly in energy than the collective mode, such that $Z^{*} \geq Z$ 
Figure 1(a) shows depolarized Raman spectra for in-plane magnetic fields ranging from 0 to 2 T. With illumination and collection along the growth axis, excitations with vanishing momentum $(q=0)$ are probed and depolarized Raman spectra, obtained with crossed incident and scattered polarizations, give access to spin flip excitations. Each spectrum shows the coexistence of a narrow low energy line with a broader high energy line, both dispersing with the applied magnetic field. Earlier ${ }^{15,20}$, we showed that the narrow line corresponds to the collective spin flip wave (SFW), the energy of which for vanishing wavevector $q$ coincides with the bare Zeeman energy $Z(B)$ [Eq. (3)]. Figure 1(b) shows that the Raman shift of the additional high energy line observed here, which is only present in depolarized spectra, increases with the applied field in the same way as $Z(B)$. We assign the high energy line to a spin flip single particle excitation (SF-SPE), the energy of which for $q=0$ is given by the renormalised Zeeman energy $Z^{*}$. The Raman response of the system exhibits both a collective and single particle response, contrary to, for example, electron spin resonance (ESR) ${ }^{21}$ which couples only with the collective response. This behavior originates from the strong resonance condition needed for RRS ${ }^{22}$.

These SF-SPE involve transitions across the Fermi level, between spin-split subbands, as illustrated in Figs. 2(c) and (d). The SF-SPE are degenerate at $q=0$ and develop into a continuum of excitations for $q \neq 0$. This continuum and the SFW dispersion, calculated from the spin-density response, ${ }^{19,20}$ are shown in Fig. 2(a). The well-defined SFW and broader SFSPE line are clearly visible in theoretical Raman spectra ${ }^{20}$, also shown in Fig. 2(a). Occupancy of the spin-up subband leads, for $q \neq 0$, to a double peak structure in the continuum spectrum, which is also observed in the experimental spectra (see e.g. Fig. 2(b)) for all samples. In summary, depolarized RRS gives access directly to both $Z$ and $Z^{*}$, the bare and the enhanced Zeeman energies, as a function of the magnetic field. 
We may define a bare spin polarization $\zeta_{0}$ to be the spin polarization of the non-interacting electron gas, such that $\zeta_{0}=m_{b} Z / 2 \pi \hbar^{2} n_{2 D}$. Then, since $\chi=\partial m_{z} / \partial b_{z}$, where the magnetization $m_{z} \propto n_{2 D} \zeta$ and $b_{z}$ is the magnetic field acting upon the 2DEG, the spin susceptibility enhancement is given by,

$$
\chi / \chi_{0}=d \zeta / d \zeta_{0}
$$

Integration of Eq.(1), combined with Eq. (4), yields the following exact result for the spin polarization enhancement:

$$
\frac{\zeta}{\zeta}=\left(1+\frac{r_{s}^{2}}{2} \frac{1}{\zeta} \frac{\partial \varepsilon_{x c}}{\partial \zeta}\right)^{-1} .
$$

If we now assume parabolic spin-split sub-bands of equal renormalized mass, such that $m_{\uparrow}^{*}=m_{\downarrow}^{*}=m^{*} \quad$ (the validity of this assumption will be discussed later), then $\zeta=m^{*} Z^{*} / 2 \pi \hbar^{2} n_{2 D}=Z^{*} / 2 E_{F}$, where $E_{F}$ is the Fermi energy of the unpolarized 2DEG. Hence:

$$
\zeta / \zeta_{0}=m^{*} Z^{*} / m_{b} Z
$$

In non-magnetic samples $Z^{*}=g^{*} \mu_{B} B$ and $Z=g_{b} \mu_{B} B$, where $\mu_{B}$ is the electron Bohr magneton. Thus, one sees from Eqs. (4) and (6) that Eq. (2), which is employed in magnetotransport investigations ${ }^{5-8,12}$, implicitly assumes a linear dependence of $Z^{*}$ on $B$, whereas Eqs. (4) and (6) are more general. Hence, we show how knowledge of both single-particle and 
collective spin flip excitation energies, and hence $Z^{*}$ and $Z$, enables the simultaneous determination of both $g^{*}$ and $g_{b}$, providing a direct determination of the g-factor enhancement.

We present in Fig. 3(a) the observed dependence of $Z^{*}$ on $Z$ determined, respectively, from the SF-SPE and SFW lines in Raman spectra (e.g, those of Fig. 1). To compare these data with numerical predictions based on Eqs. (5) and (6) we have determined the electron density $n_{2 D}$ from measurements of polarized Raman spectra, measured with parallel incident and scattered polarizations and zero applied magnetic field. Under strong resonance the polarized Raman spectrum reveals unscreened single-particle excitations (SPE). The dispersion of the high-energy cut-off of the SPE band follows $\hbar v_{F} q$, where $v_{F}$ is the Fermi velocity of the nonpolarized 2DEG. Table I gives the Fermi velocities measured by this technique. The electron density is then given by $n_{2 D}=\left(m_{F}^{*} v_{F} / \hbar\right)^{1 / 2} / 2 \pi$, where $m_{F}^{*}$ is the Fermi electron mass. Renormalization of the Fermi-mass has been predicted $^{23}$ and confirmed by transport measurements ${ }^{8,12}$ in a single heterojunction, for which case $\left(r_{s} \sim 2\right)$ the out of plane extension of the $2 \mathrm{D}$ electron wavefunction is much larger than the quantum well thickness of the present samples. Thus, an evaluation of $m_{F}^{*}$ for our samples is not available and so we give, in Table I, the non-corrected density $n_{2 D}^{0}$ and corresponding $r_{s}^{0}$, determined assuming $m_{F}^{*}=m_{b}=0.105 m_{e}$.

We present in Fig. 3(b) the Zeeman energy enhancement $Z^{*} / Z$, deduced from Fig. 3(a), as a function of $\zeta_{0}$, which is determined assuming $n_{2 D}=n_{2 D}^{0}$ (this is expected to give a correction of less than 5\% in $r_{s}$ ). This is compared with the calculated dependence on $\zeta_{0}$ of the spin polarization enhancement $\zeta / \zeta_{0}$, determined from Eq. (5) using the exchange-correlation 
energy $\varepsilon_{x c}$ from Ref. 2 and accounting for finite thickness effects using Ref. 3. Fig. 3(b) demonstrates that $Z^{*} / Z$ and $\zeta / \zeta_{0}$ are not proportional, as expected from Eq. (6). This suggests that for finite spin polarization we must consider the mass $m^{*}$ in Eq. (6) to be a spin polarization dependent renormalized mass, $\bar{m}^{*}$.

Figure 3(c) shows $\bar{m}^{*} / m_{b}$ obtained by division of the theoretical $\zeta / \zeta_{0}$ by the experimental $Z^{*} / Z$, as a function of the spin polarization $\zeta$ (determined from the dependence of $\zeta / \zeta_{0}$ with $\left.\zeta_{0}\right)$. We find an enhanced mass $\bar{m}^{*}\left(\bar{m}^{*} / m_{b} \geq 1\right)$ for high $\zeta$, and a strong variation with the spin polarization. Such behavior cannot be understood within the assumption of equal masses $\left(m_{\uparrow}^{*}=m_{\downarrow}^{*}=m^{*}\right)$. This is consistent with a recent prediction of strong non-linear behavior of $m_{\uparrow}^{*}$ and $m_{\downarrow}^{*}$ with $\zeta$, which gives $m_{\uparrow}^{*} / m_{\downarrow}^{*} \approx 1.1$ for $\zeta=50 \%$.

To understand $\bar{m}^{*} / m_{b}$ requires the derivation of Eq. (6) with the inclusion of a spin-dependent renormalization of the mass. If we assume the bands are still parabolic, but $m_{\uparrow}^{*} \neq m_{\downarrow}^{*}$, we find

$$
\bar{m}^{*}=\frac{2 m_{\uparrow}^{*} m_{\downarrow}^{*}}{m_{\uparrow}^{*}+m_{\downarrow}^{*}} \frac{1}{1-\zeta^{-1}\left(m_{\uparrow}^{*}-m_{\downarrow}^{*}\right) /\left(m_{\uparrow}^{*}+m_{\downarrow}^{*}\right)} .
$$

We present in Fig. 3(c) $\bar{m}^{*} / m_{b}$ determined using Eq. (7) with Fermi masses given by Ref. 13 (Z\&D). Although this calculation neglects correlations and thickness corrections, it predicts a mass enhancement of the same order as we observe, as well as a dependence on the spin polarization. Nevertheless, the strong increase of $\bar{m}^{*} / m_{b}$ with spin polarization is not reproduced, and it is likely that a non-parabolic mass enhancement is required to account for 
the observed dependence of the mass enhancement in Fig 3(c). For this, the spin resolved electron self-energy and density of states are required for a derivation of $\bar{m}^{*} / m_{b}$.

We have presented resonant Raman scattering measurements of the zero momentum spin flip excitations in a spin-polarized 2DEG embedded in a high mobility semi-magnetic quantum well. The spin flip of a single particle coexists with the spin flip wave in Raman scattering spectra. Using this, we have explored the dependence of the spin susceptibility, g-factor and mass enhancement over a wide range of spin-polarization, without the effects of Landau quantization. We provide a direct determination of the g-factor enhancement and we have introduced a generalized expression (Eqs. (6) and (7)) of the spin polarization degree, necessary for such high spin polarization. We introduce an average renormalized mass where both the zone centre mass and the Fermi mass renormalization play a role. We give new data about the spin polarization degree dependence of this average mass and compare it with the only available theory. The latter does not well reproduce the data, indicating a need for the development of an accurate spin resolved theory for self-energy and mass renormalization.

We thank the UK EPSRC and CNRS for financial support and S. De Palo, P. Gori-Giorgi, M. Polini, G. F. Giuliani and G. Vignale for fruitful discussions. 
Table I. Sample parameters (see text).

\begin{tabular}{|c|c|c|c|c|}
\hline Sample & (A) & (B) & (C) & (D) \\
\hline$x(\%)$ & 0.75 & 0.84 & 0.78 & 0.79 \\
\hline$v_{F}\left(10^{6} \mathrm{~cm} / \mathrm{s}\right)$ & 14.7 & 13.4 & 13.3 & 12.7 \\
\hline$n_{2 D}^{0}\left(10^{11} \mathrm{~cm}^{-2}\right)\left[r_{s}^{0}\right]$ & $2.83[2.11]$ & $2.35[2.31]$ & $2.31[2.33]$ & $2.12[2.47]$ \\
\hline
\end{tabular}




\section{Figure Captions}

FIG.1 (a) (Color online) Typical depolarized Raman spectra of one of the four samples studied (sample A), measured at $E_{i}=1.621 \mathrm{eV}$, showing the spin flip wave (SFW) and the spin flip single particle excitation (SF-SPE). The background at high Raman shift is from luminescence. The Voigt back scattering geometry $[x(\sigma \pi) \bar{x}]$ is parallel to the growth axis $x$ and perpendicular to the in-plane field. (b) Magnetic field dependence of energies $Z$ and $Z^{*}$ deduced from (a). $Z(B)$ has been fitted with the Brillouin function.

FIG.2 (a) (Color online) Typical theoretical dispersions of spin flip excitations of the SP2DEG calculated for $\zeta=-0.4$ and $r_{\mathrm{s}}=2.0$. Full circles are the SFW and the hatched area is the SF-SPE continuum. Lines numbered 1, 2, 3, 4 correspond respectively to the excitations 1, 2, 3, 4 in (d): Line 4 (1) is the excitation of a spin down electron with initial wavector $k=k_{F \downarrow}(k$ $=-k_{F} \downarrow$ ). Lines 2 and 3 are limits where the number of excitations is restricted due to filling of the up spin subband. Overlaid are the calculated Raman spectra for $q / k_{\mathrm{F}}=0$ and 0.2 .. (b) Experimental spectra obtained on sample A for $B=4.8 \mathrm{~T}$ and $q=0$ and $9.5 \mu \mathrm{m}^{-1}$ $\left(q / k_{F} \approx 0.1\right)$. (c) and (d) Schematic of spin-split subbands indicating representative SF-SPE excitations for (c) $q=0$ and (d) non-zero $q$. 
FIG. 3 (a) (Color online) $Z^{*}(Z)$ for all samples. (b) Comparison of $Z^{*} / Z$ (symbols) deduced from (a) and theoretical $\zeta / \zeta_{0}$ (lines) given by Eq. (5) as a function of the bare spin polarization $\zeta_{0}$. Line colors match with symbol colors for the different samples. The black dotted lines around the solid black line for sample A are limits of $\zeta / \zeta_{0}$ when including the error on $r_{s}^{0}$ and varying the thickness of the square well from $\pm 10 \%$ (a variation which overestimates the error of the growth technique and any error from neglecting the wavefunction penetration into the barrier). (c) Symbols indicate the average renormalized mass $\bar{m}^{*}(\zeta) / m_{b}$ extracted from (b) as a function of the spin polarization $\zeta$, determined from $\zeta_{0}$ using $\zeta / \zeta_{0}$. Lines are Z\&D prediction (Ref. 13) for $r_{s}=1,2$ and 3. We have added $m_{F}^{*}(\zeta=0)$ from Ref. 23. Sample C has been removed for clarity. 
Figures

\section{FIG.1}

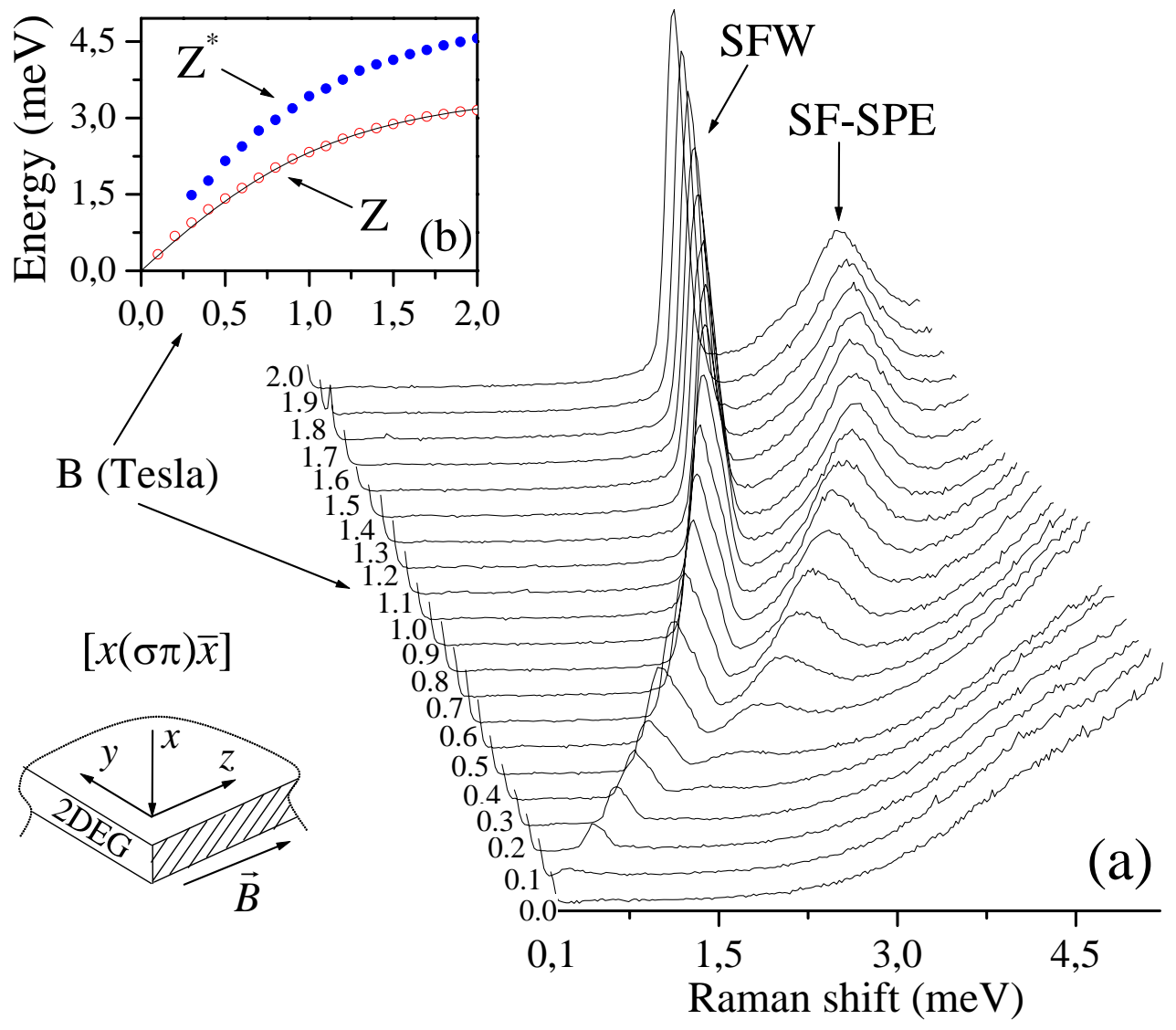




\section{FIG.2}
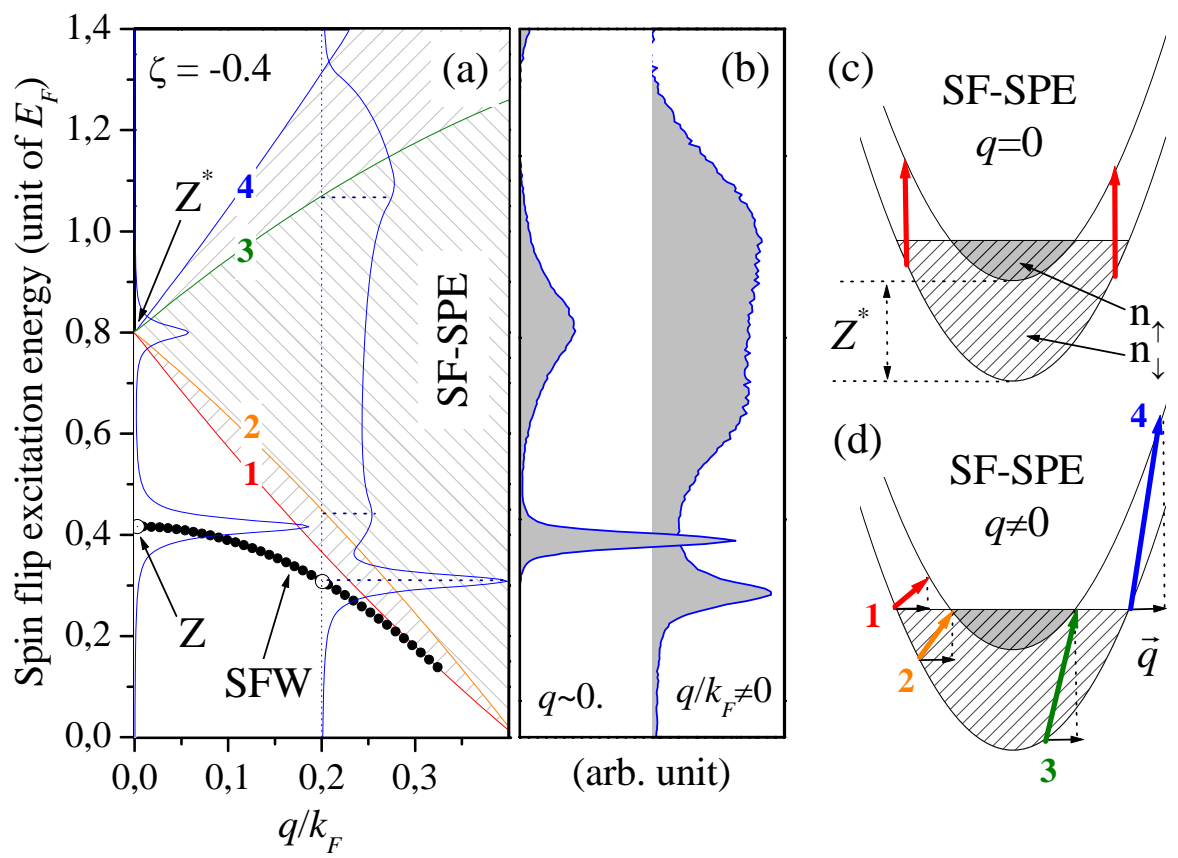
FIG.3
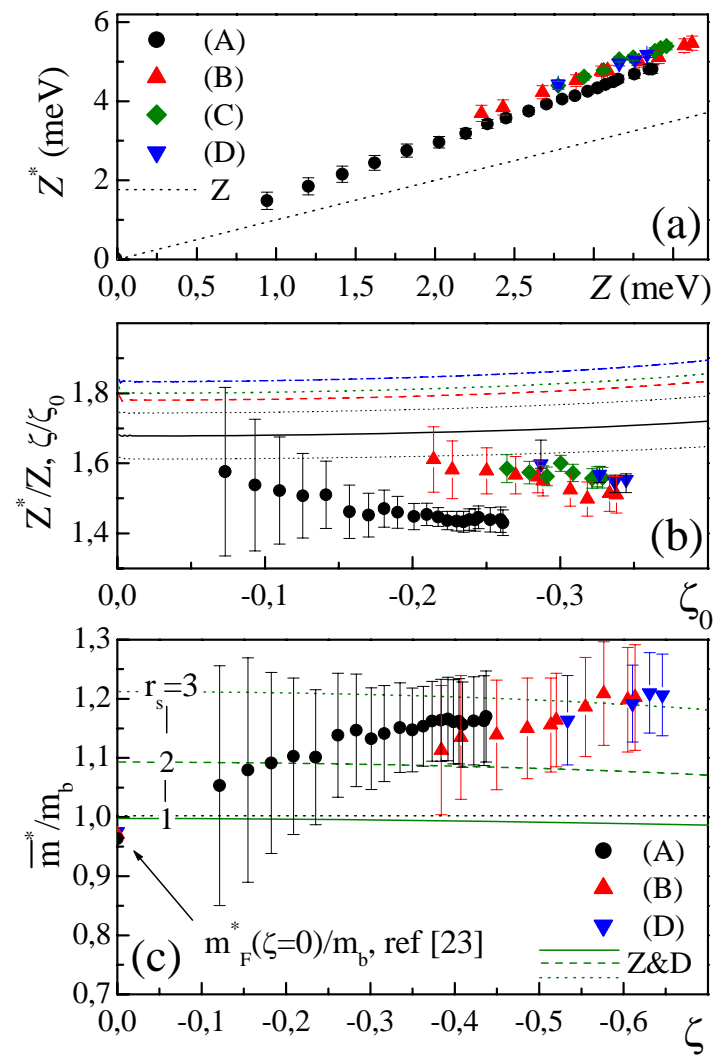


\section{References}

1 D. R. Hamann and A. W. Overhauser, Phys. Rev. 143, 183 (1966).

${ }^{2}$ C. Attaccalite et al., Phys. Rev. Lett. 88, 256601 (2002).

${ }^{3}$ S. De Palo et al., Phys. Rev. Lett. 94, 226405 (2005).

${ }^{4}$ Y. Zhang and S. Das Sarma, Phys. Rev. Lett. 96, 196602 (2006).

${ }^{5}$ E. Tutuc, S. Melinte, M. Shayegan, Phys. Rev. Lett. 88, 36805 (2002).

${ }^{6}$ J. Zhu et al., Phys. Rev. Lett. 90, 56805 (2003).

${ }^{7}$ T. Okamoto et al., Phys. Rev. Lett. 82, 3875 (1999); K. Vakili et al., Phys. Rev. Lett. 92, 226401 (2004); A. Ghosh et al., Phys. Rev. Lett. 92, 116601 (2004).

${ }^{8}$ V. M. Pudalov et al., Phys. Rev. Lett. 88, 196404 (2002).

${ }^{9}$ A. A. Shashkin et al., Phys. Rev. Lett. 96, 36403 (2006)

10 S. H. Vosko, J. P. Perdew, Can. J. Phys. 53, 1385 (1975).

${ }^{11}$ in CdTe, $a_{B}^{*} \approx 5.3 \mathrm{~nm}, R_{y}^{*}=\hbar^{2} / 2 m^{*}\left(a_{B}^{*}\right)^{2} \approx 14.3 \mathrm{meV}$

${ }^{12}$ Y. W. Tan et al., Phys. Rev. Lett. 94, 16405 (2005).

${ }^{13}$ Y. Zhang and S. Das Sarma, Phys. Rev. Lett. 95, 256603 (2005).

14 A. Pinczuk et al., Phys. Rev. Lett. 68, 3623 (1992).

${ }^{15}$ B. Jusserand et al., Phys. Rev. Lett. 91, 86802 (2003).

16 S. Oelting, Surf. Sci. 196, 273 (1988).

${ }^{17}$ J. A. Gaj, R. Planel, G. Fishman, Solid State Commun. 29, 435 (1979).

${ }^{18}$ L. D. Landau and E. M. Lifshitz, The Classical Theory of Fields, 2nd edition (Pergamon Press, Oxford, 1962), Chap. 5, p.120.

19 A. K. Rajagopal, Phys. Rev. B 17, 2980 (1978).

${ }^{20}$ F. Perez et al., Phys. Status Solidi B 243, 873 (2006).

${ }^{21}$ D. Stein, K. v. Klitzing, and G. Weimann, Phys. Rev. Lett. 51, 130 (1983). 
22 D. W. Wang and S. Das Sarma, Phys. Rev. B 65, 125322 (2002).

${ }^{23}$ R. Asgari et al., Phys. Rev. B 71, 45323 (2005). 